\title{
Nonsense-mediated mRNA decay mutes the splicing defects of spliceosome component mutations
}

\author{
TADASHI KAWASHIMA, ${ }^{1}$ MATTEO PELLEGRINI, ${ }^{2}$ and GUILLAUME F. CHANFREAU ${ }^{1}$ \\ ${ }^{1}$ Department of Chemistry and Biochemistry and the Molecular Biology Institute, University of California at Los Angeles, \\ Los Angeles, California 90095-1569, USA \\ ${ }^{2}$ Department of Molecular, Cellular and Developmental Biology, University of California at Los Angeles, Los Angeles, \\ California 90095-1606, USA
}

\begin{abstract}
The role of many splicing factors in pre-mRNA splicing and the involvement of these factors in the processing of specific transcripts have often been defined through the analysis of loss-of-function mutants in vivo. Here we show that inactivating the nonsense-mediated mRNA decay (NMD) results in an enhancement of splicing phenotypes associated with several $S$. cerevisiae

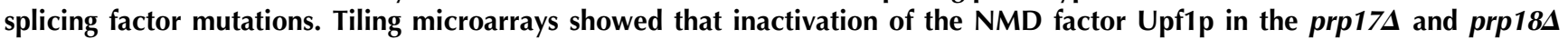
mutant strains results in a larger spectrum of splicing defects than what is observed in the single mutants, including new transcripts previously shown unaffected by Prp17p or Prp18p inactivation. Inactivation of Upf1p in the second step/recycling factor prp22-1 mutant and in the nam8s and mud1s U1 snRNP component mutants also increase unspliced precursor accumulation of several specific transcripts. In addition, deletion of UPF1 partially suppresses the growth defects associated with the prp17A or prp22-1 mutations, demonstrating a positive genetic interaction between NMD and splicing factor mutants. These results show that RNA surveillance by NMD can mask some of the effects of splicing factor mutations, and that the roles of splicing factors cannot be fully understood in vivo unless RNA degradation systems that degrade unspliced precursors are also inactivated.
\end{abstract}

Keywords: NMD; splicing; yeast; RNA degradation; quality control

\section{INTRODUCTION}

Nonsense-mediated mRNA decay (NMD) is an RNA degradation mechanism that degrades pre-mRNA containing premature translation termination codons (PTCs) (BehmAnsmant et al. 2007; Chang et al. 2007; Isken and Maquat 2008). Due to the high probability of encountering a PTC, unspliced pre-mRNAs or some alternatively spliced RNAs are potential targets for NMD. Indeed, NMD is involved in regulating highly conserved alternatively spliced forms containing PTCs (Lareau et al. 2007; Ni et al. 2007) and degrades unspliced precursors of transcripts spliced with suboptimal efficiency in C. elegans (Mitrovich and Anderson 2000), S. cerevisiae (He et al. 1993; Sayani et al. 2008), and Paramecium (Jaillon et al. 2008). These studies led to the idea that NMD might act as a general quality control

Reprint requests to: Guillaume F. Chanfreau, Department of Chemistry and Biochemistry and the Molecular Biology Institute, University of California at Los Angeles, Los Angeles, CA 90095-1569, USA; e-mail: guillom@chem.ucla.edu; fax: (310) 206-4038.

Article published online ahead of print. Article and publication date are at http://www.rnajournal.org/cgi/doi/10.1261/rna.1736809. mechanism for defective or suboptimal splicing. Based on this, one might expect NMD to be also involved in degrading unspliced precursors generated by mutations of splicing signals or by inactivation of splicing factors. Surprisingly, combining a thermosensitive mutation of the Prp2p splicing factor with NMD inactivation did not result in the stabilization of several unspliced precursors (Bousquet-Antonelli et al. 2000). NMD integrity also does not affect the steady-state levels or the rate of decay of unspliced precursors of splicing substrates containing a mutated actin intron (Hilleren and Parker 2003; Sayani et al. 2008). In addition, several other unspliced precursors resulting from splicing signal mutations accumulate to high levels in the presence of active NMD (Vijayraghavan et al. 1986; Chanfreau et al. 1994; Sayani et al. 2008). These observations led to the conclusion that NMD does not contribute to the quality control of splicing in splicing mutants. However, the recent observation that NMD degrades most unspliced precursors resulting from a $5^{\prime}$-splice site mutation in the RPS10B gene (Sayani et al. 2008) led us to investigate whether NMD can mask or reduce the effects of trans-acting splicing factor mutations. In this study, we 
show that many transcripts do not exhibit any detectable splicing defect in several spliceosome component mutants unless NMD is also disrupted. In addition we also show that the quantitative effects of these splicing mutants are generally enhanced in the absence of functional NMD. These results reveal that NMD can mask the effects of splicing factor inactivation by degrading unspliced RNAs that accumulate due to defective splicing. Our observations show that the full extent of the role of splicing factors cannot be fully understood in vivo unless RNA degradation systems that eliminate these unspliced RNAs are inactivated as well.

\section{RESULTS}

\section{NMD quantitatively reduces the splicing defects associated with inactivation of the Prp17p and Prp18p splicing factors}

To investigate whether NMD can mask the effects of splicing factor mutations in vivo, we first inactivated the NMD factor Upflp in yeast strains carrying deletions of the nonessential splicing factors Prp17p and Prp18p. The choice of these nonessential splicing factors was based on the fact that unspliced RNA levels could be analyzed at steadystate and normal growth temperatures without having to shift the cells to nonpermissive temperatures, alleviating the issues of viability and mild heat-shock conditions. We constructed the double

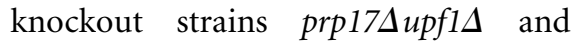
prp18supf1 1 by direct knockout of UPF1 in the prp17A or prp18A backgrounds. We then performed tiling array analysis of RNAs and analyzed the intronic signals in all single and double mutants strains. To investigate the extent to which NMD can mask splicing defects in the prp17s and $\operatorname{prp} 18 \Delta$ strains, we generated Z-scores based on measuring the ratio of intronic signals between each strain (provided in Supplemental Table 1). Our previous study had shown that analysis of the variations in intronic signals is a valid first approximation of the amount of unspliced precursor that accumulates in mutant strains compared with the wild type (Sayani et al. 2008). Intronic $Z$ scores were based on the average of the $\log 2$ of the ratio of the signals obtained from probes located in introns or spanning the exon-intron junctions between each of the strains analyzed. Because the number of probes varied depending on introns, the use of the $\mathrm{Z}$-scores allowed us to take into account the number of probes used to measure the signal from each intron. Comparison of the Z-scores between the prp17 $\Delta$ mutant and the wild type (Fig. 1A, $x$-axis), and between the prp17 $\Delta$ upf $1 \Delta$ and the upfis mutant (Fig. 1A, $y$-axis) showed that the quantitative effect of the prp17 $\Delta$ deletion on intronic signal is much more pronounced when NMD is inactive (Fig. 1A, left panel). This is illustrated by the large number of introns that fall above the diagonal line, or for which the Z-score is close to or lower than zero for the prp17A mutant to the wild-type comparison (Fig. 1A, $x$-axis), but which exhibit an increase of Z-score above zero in the double mutant (Fig. 1A, $y$-axis). The same effect was observed when comparing the effect of NMD inactivation (Fig. 1A, right panel, upf1 $1 \Delta$ versus WT) to the effects of NMD inactivation in the context of the prp17A deletion (Fig. 1A, right panel,
A
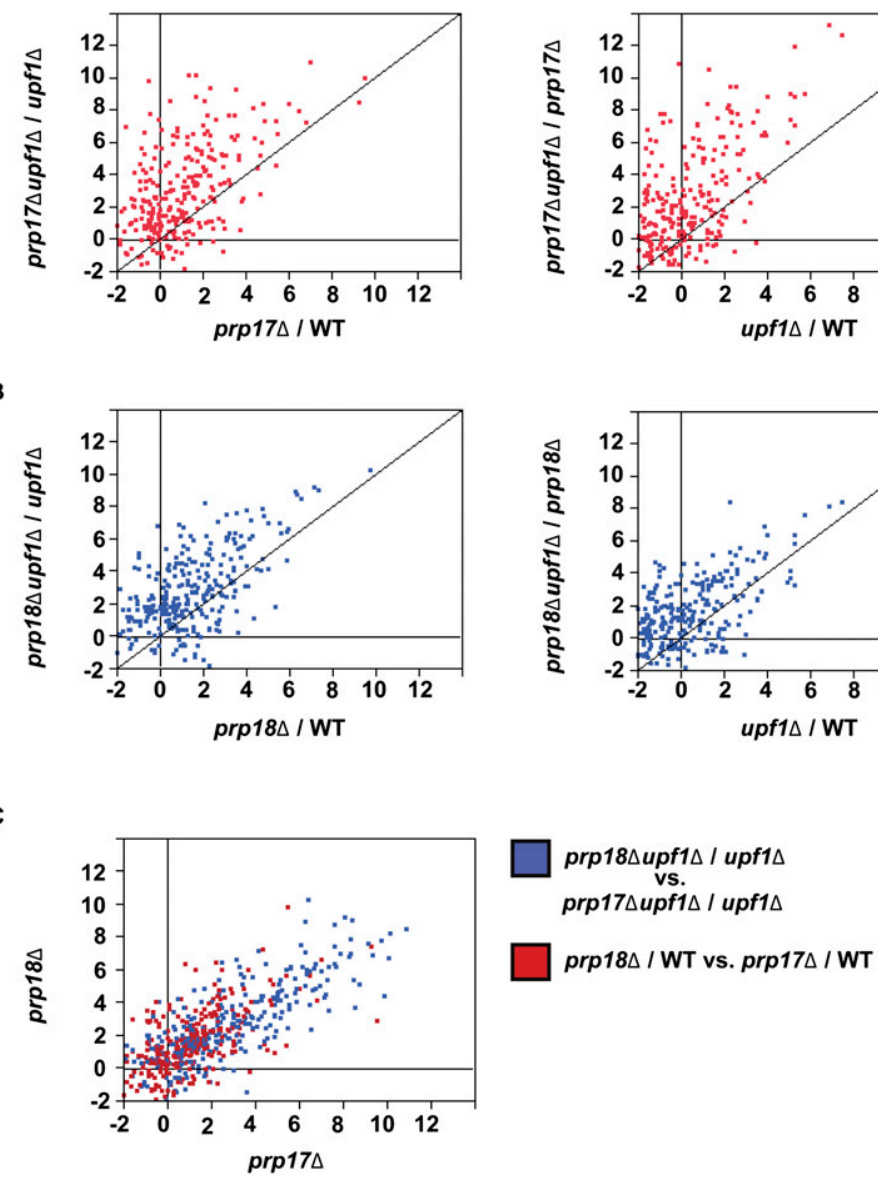

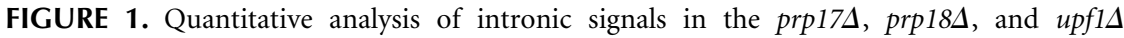
mutants and in double mutants. Plotted are the Z-scores for the average of the $\log 2$ of the ratio of intronic signals for all introns of the $S$. cerevisiae genome in the indicated strain comparisons. Lines indicate the diagonal on which equal Z-score for the strain comparisons would be located. 


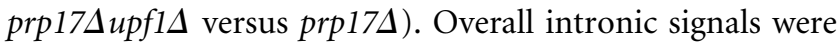
generally higher in the prp $17 \Delta u p f 1 \Delta$ double deletion strain than in either single mutant, which shows that NMD quantitatively reduces intronic signals in the prp17d deletion mutant. The same effect was observed for the prp18s upf1 $1 \Delta$ double mutant when compared with either prp18s or upflA single mutants (Fig. 1B).

To provide an independent way to visualize the effect of

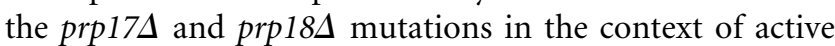
or inactive NMD, we plotted the intronic scores of the comparison sets of these mutants against each other in the context of active NMD (Fig. 1C, red dots, each single mutant is compared with wild type) or inactive NMD (blue dots; each double mutant is compared with the upfis single mutant). When plotted on the same graph, the data points observed in the context of inactive NMD (Fig. 1C, blue dots) are shifted to higher values than those observed

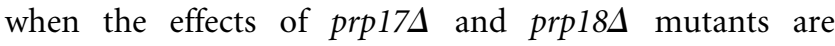
compared when NMD is active (Fig. 1C, red dots). This observation shows that the deletion of PRP17 or PRP18 results in higher intronic signals when these mutations are combined with NMD inactivation. We note that the effects of Prp17p and Prp18p inactivation are generally well correlated, whether NMD is active or not (Fig. 1C), with only a small minority of introns showing differential effects upon inactivation of Prp17p or Prp18p. Overall, these data show that NMD reduces intronic signal accumulation in splicing factor mutants when analyzing the entire intronic population of S. cerevisiae. Even if a fraction of the intronic signal comes from lariat intermediates generated by inactivation of these second step splicing factors, these results suggest that a significant fraction of the unspliced precursors generated by spliceosome component mutations are degraded by NMD, which limits or prevents their accumulation in vivo.

\section{NMD mutes transcript-specific splicing defects associated with inactivation of Prp17p and Prp18p}

The previous analysis revealed that inactivation of Upflp in

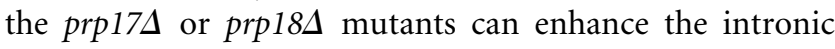
signal accumulation phenotype associated with these splicing mutants, suggesting that the splicing defects of these mutants can be more readily detected or is exacerbated when NMD is inactivated. We next sought to identify transcripts for which NMD would completely mask the effects of the Prp17p or Prp18p depletion. Such transcripts would exhibit no increase of intronic signal in the prp17A and prp18s mutants compared with wild type, but a large signal increase in the double mutants when NMD is also inactivated (Fig. 1A,B, upper left quadrants). To facilitate visualization of these transcripts, we performed hierarchical clustering analysis based on the intronic Z-scores and searched for transcripts with no intronic signal accumula-

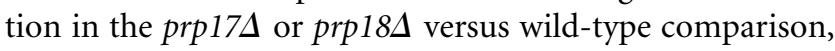

but for which an increase of intronic signal was detected in the double-mutant strains when compared with each single mutant (Figs. 2, 3). This analysis revealed several transcripts for which intronic signal increase is not detectable in either of the single mutants, but for which a strong increase in intronic signal can be detected when comparing

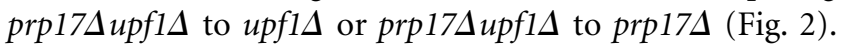
Similar observations were made for the prp184-derived mutants, although the number of introns affected was smaller than for prp174 (Fig. 3). The intronic signal increase observed for many introns does not result from a general splicing defect in the double mutants, since several precursors did not show an increase of intronic signal in either the single or double mutants (Figs. 2, 3, Clusters A). The lack of effect observed for these transcripts was not due to the absence of premature termination codons (PTCs), as the large majority of yeast unspliced pre-mRNAs contain PTCs, as shown previously (Sayani et al. 2008). Interestingly, this analysis showed that the absence of Prp17p can result in intronic signal increase for short introns, in contrast to what previous analyses had shown (Clark et al. 2002; Sapra et al. 2004). For example, the BOS1, CIN2, and MTR2 genes showed an increase of intronic signal

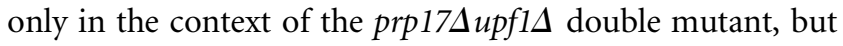

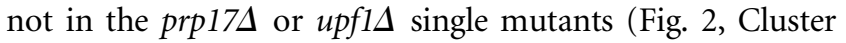
B). The small sizes of the BOS1, CIN2, and MTR2 introns (73, 80, and 99 nucleotides [nt], respectively) show that Prp17p can affect the splicing of introns shorter than $100 \mathrm{nt}$, but that this effect is masked by NMD. We also observed a strong increase of intronic signal in double mutants for a large number of ribosomal protein genes (Figs. 2, 3, Clusters B,C,E,F). These genes were not found to accumulate unspliced precursors in NMD mutants in a normal splicing background (Sayani et al. 2008). These results show that NMD can affect a larger number of transcripts than previously estimated. However, in the absence of splicing mutations, the splicing of these transcripts is robust enough to prevent escape of unspliced precursors from the spliceosome.

The previous analyses measured intronic signal accumulation; therefore, it was possible that the increase of signal observed in the double mutants may be indicative of accumulation of RNA species other than the unspliced precursors, such as lariat intermediates that may result from the inactivation of the Prp17p and Prp18p second step splicing factors. To investigate whether or not the increase of signal observed was due to unspliced precursor stabilization, we first performed Northern blot analysis on the RPL16A, RPS13, and RPS11B transcripts (Fig. 4A). Based on the tiling array measurements, RPL16A and $R P S 11 B$ were predicted to have stronger precursor accumulation in the double mutants than each of the corresponding single mutants, while unspliced precursor accumulation for RPS13 was predicted to be found in the upf1A strain and not exacerbated by deletion of Prp17p or 


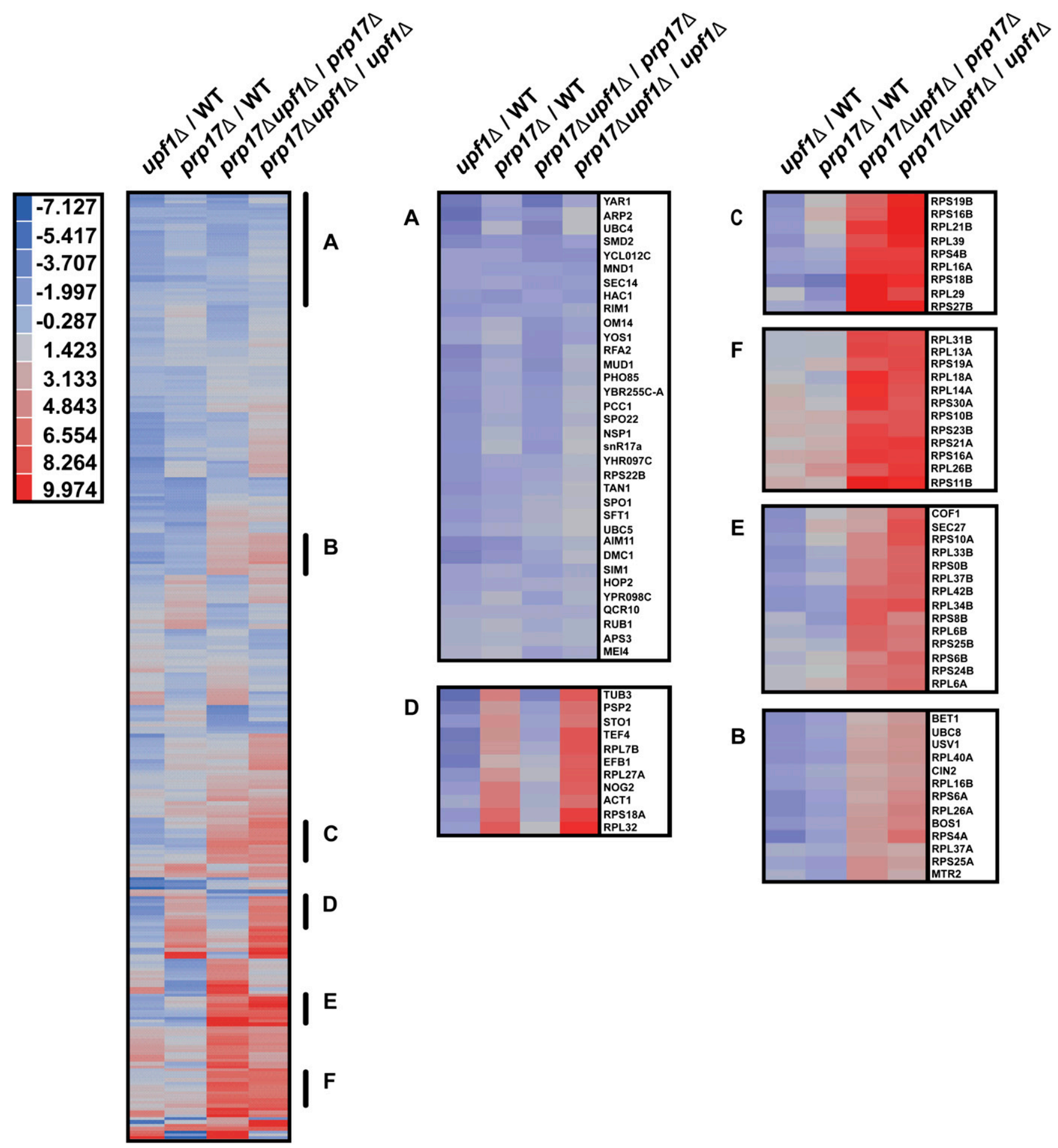

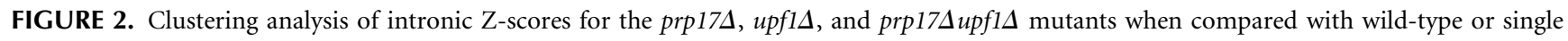
mutants.

Prp18p. Northern analysis validated the prediction made by the tiling array analysis, with a dramatic accumulation

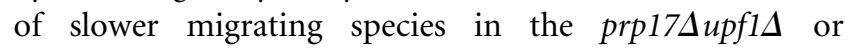

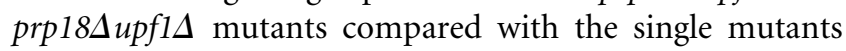
for RPL16A and RPS11B. This was not observed for RPS13, for which the accumulation of the slower migrating species was not increased by disruption of Prp17p or Prp18p. We also observed slightly faster migrating species that are detectable in the prp174 or prp184 single mutants that might correspond to intron-exon2 lariat intermediates 


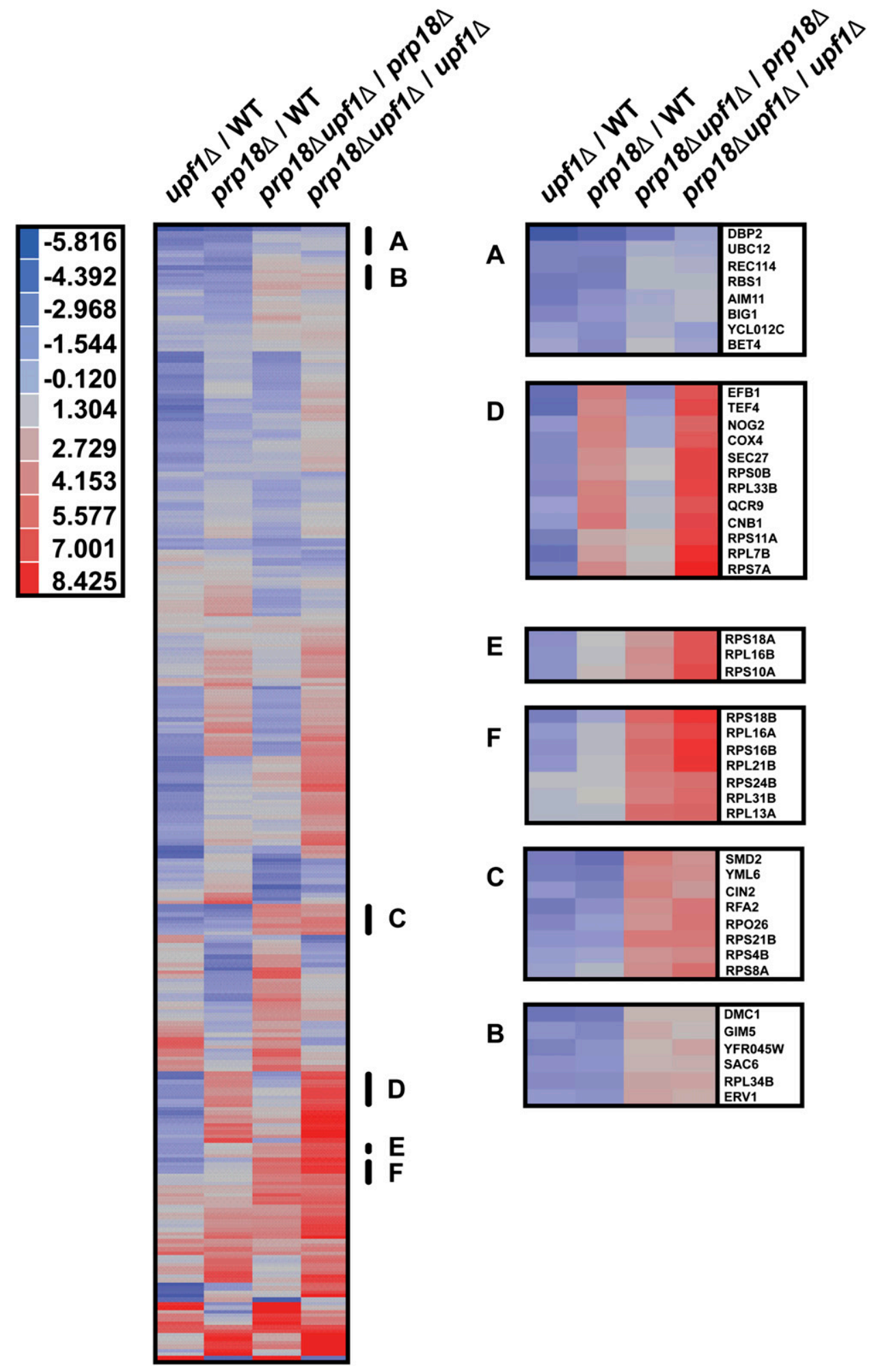

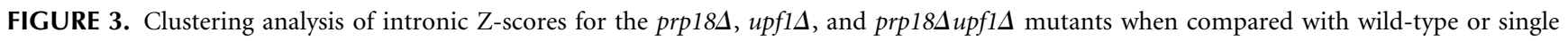
mutants. 
A

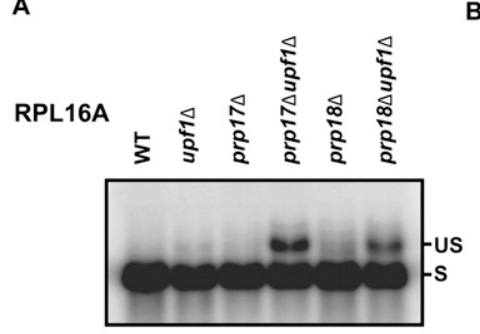

RPS11B

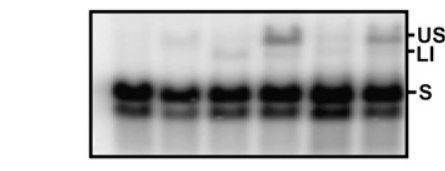

RPS13

B
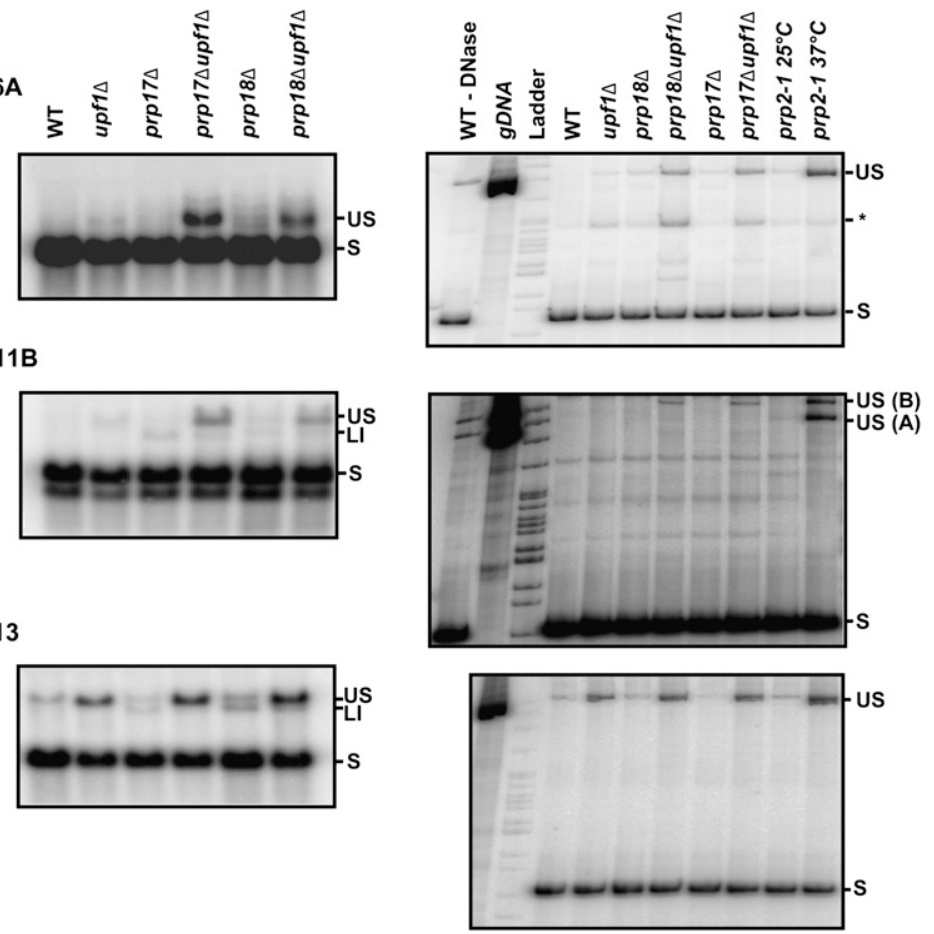

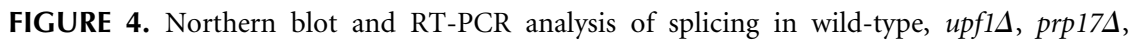

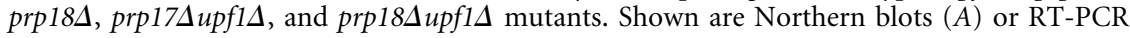
$(B)$ analysis of the indicated mRNAs (RPL16A, RPS11B, RPS13) in the corresponding strains. For the RT-PCR analysis, a genomic DNA sample and RNAs extracted from a prp2-1 strain grown at $25^{\circ} \mathrm{C}$ or shifted for $30 \mathrm{~min}$ to $37^{\circ} \mathrm{C}$ were included as positive controls for the detection of unspliced species. The migration of the unspliced pre-mRNAs and spliced mRNAs are indicated by US and S, respectively. LI indicates the lariat intron-exon2 intermediates detected by Northern blot in the prp17A, prp18A mutants. The species labeled * for RPL16A were not mapped and might correspond to a cryptic splicing event. For the RPS11 RT-PCR panel, the oligonucleotides used did not discriminate between the RPS11A and RPS11B copies; therefore, both unspliced species are amplified by PCR.

(Fig. 4A, LI). However, their accumulation is not exacerbated by Upflp inactivation in contrast to the presumed unspliced precursors (Fig. 4A). This is in agreement with previous studies showing that lariat intermediates are degraded in the cytoplasm in a Dbr1p-dependent manner (Hilleren and Parker 2003). This Northern analysis suggested that unspliced precursor accumulation of RPS11B and $R P L 16 A$ was exacerbated in the prp17Aupf1A or

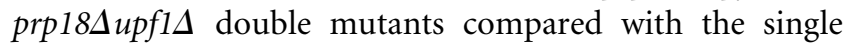
mutants. However, due to the small size of exon 1 , we could not rule out that some of these species postulated to be unspliced precursors may actually correspond to lariat intermediates. To further investigate this possibility we performed RT-PCR on DNase-treated RNA samples from these strains using oligonucleotides hybridizing to exon 1 and exon 2 sequences. These oligonucleotides specifically amplify mRNAs and unspliced precursors, allowing their detection independently from lariat intermediates (Fig. $4 \mathrm{~B})$. We also included in this analysis samples extracted from the prp2-1 splicing mutant grown at $25^{\circ} \mathrm{C}$ or shifted for $30 \mathrm{~min}$ at $37^{\circ} \mathrm{C}$ (Fig. $4 \mathrm{~B}$, last two lanes) to serve as positive controls for the accumulation of unspliced precursors, as well as a PCR reaction performed on genomic DNA (gDNA) or on wild-type RNAs that were not treated with DNase (Fig. $4 \mathrm{~B}$, first two lanes for RPL16A and $R P S 11 B)$. This analysis showed that unspliced (US) precursors of RPL16A and $R P S 11 B$ accumulate specifically in

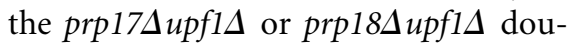
ble mutants, in contrast to single mutants in which these species are barely visible or undetectable. Thus, the slower migrating species detected on Northern blots in Figure 4A, those that accumulate specifically in the double mutants, correspond to unspliced precursors. We note that the accumulation of un-

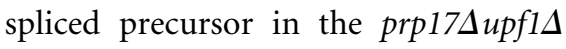
or prp18 1 upf1 $1 \Delta$ double mutants is specific to $R P S 11 B$, as unspliced species of RPS11A cannot be detected in these strains. This is in contrast with the prp2-1 mutant, in which both RPS11A and $R P S 11 B$ unspliced precursors can be detected (Fig. 4B, middle panel). Finally, analysis of RPS13 by RT-PCR confirmed the observation made by Northern blot that the accumulation of unspliced precursors of RPS13 in the upfls mutant is not exacerbated by inactivation of Prp17p or Prp18p (Fig. $4 \mathrm{~B}$, bottom panel).

\section{RT-PCR analyses show that the effect of NMD in masking the effects of splicing defects is more widespread than revealed by tiling arrays}

Even when considering the RT-PCR data described above, some of the intronic signals detected by tiling arrays may result either from unspliced precursors or from lariat species such as intron-exon2 intermediates

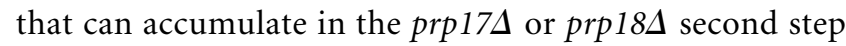
mutants. For some of the intron-containing genes, we considered the possibility that we did not detect any

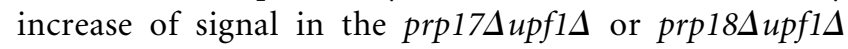
double mutants compared with the single splicing mutants, because the signal coming from lariat intermediates

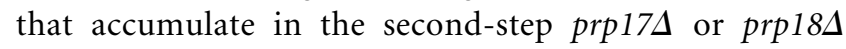
mutants exceeds the signal resulting from unspliced precursors in the double mutants. To extend our studies, we analyzed by RT-PCR unspliced precursor accumulation for five additional transcripts, TUB1, TAF14, LSB3, $A C T 1$, and $M E R 2$, which exhibited a wide range of intronic $\mathrm{Z}$-scores as determined by the tiling arrays 
(Supplemental Table 1). Based on the Z-scores, the

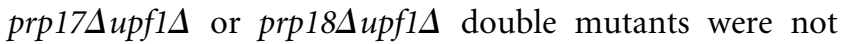
predicted to exhibit higher unspliced precursor accumulation than the single prp $17 \Delta$ or prp $18 \Delta$ mutants for TUB1, LSB3, and TAF14. However RT-PCR analysis showed that unspliced precursor accumulation is exacerbated for

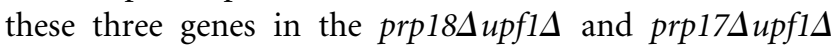
double mutants compared with each single mutant (Fig. $5)$. In contrast, actin (ACT1) precursors were undetectable

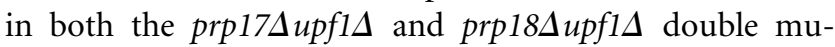
tants, while these species could be detected in the prp2-1 mutant. In addition, a meiotic transcript which naturally accumulates unspliced pre-mRNAs in vegetative conditions such as MER2 was also unaffected by NMD inactivation, either alone or in combination with splicing mutants (Fig. 5). This result corroborates previous observations that unspliced precursors of meiotic transcripts are not subject to NMD, possibly because of nuclear retention (Scherrer and Spingola 2006). We conclude that the tiling array analysis provided us with a minimal list of genes for which unspliced precursor accumulation is exacerbated by in-

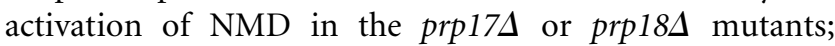
however, the effects are likely to be more widespread than found using the arrays, because the intronic signal that results from lariat intermediate accumulation in the

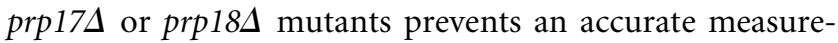

ment of the accumulation of unspliced precursors for a subset of intron-containing transcripts.

\section{NMD reduces the amount of several unspliced precursors in the prp22-1, nam8s, and mud1s splicing mutants}

The previous analysis showed that inactivation of Upflp can enhance or reveal unspliced precursor accumulation phenotypes associated with deletion of the spliceosome components Prp17p or Prp18p by repressing the degradation of some of the precursors normally targeted by NMD. To extend these results to other splicing mutants, we combined the prp22-1 thermosensitive mutant (Vijayraghavan et al. 1989) and the U1 snRNP components nam8s (Gottschalk et al. 1998) or mud1s (Liao et al. 1993) deletion mutants with the upf1s deletion by direct knockout of UPF1 in the corresponding single mutants. We grew the prp22-1 upf1s mutant strain and corresponding single mutants at 25 or $30^{\circ} \mathrm{C}$ to investigate whether inactivation of Upflp could reveal or enhance some splicing defects in the prp22-1 mutant, even at permissive temperatures. This approach allowed us to avoid shifting the cells to nonpermissive temperature, because such a shift might induce growth arrest in the prp22-1 mutant, with possible interference with the NMD process. We then investigated by RT-PCR the accumulation of unspliced transcripts for the same subset of transcripts previously analyzed for the prp17A or prp18 mutants series (Fig. 6). This analysis showed that the level of unspliced RPL16A, RPS11B, and TAF14, precursors detected by RT-PCR was slightly higher in the prp22-1 mutant grown at $30^{\circ} \mathrm{C}$, but was exacerbated when the upfls deletion was combined with this mutation. This effect was specific to these transcripts, as it was not observed for the RPS13, LSB3, MER2, TUB1, and ACT1 genes (Fig. 6). In contrast, we did not detect exacerbation of unspliced precursor accumulation when the prp22-1 and prp22-1 upf1s mutants were grown at $25^{\circ} \mathrm{C}$ (Fig. 6). It is possible that the $\mathrm{NMD}$ pathway is less active at this lower temperature, or that the $30^{\circ} \mathrm{C}$ temperature used previously exacerbates the degradation of unspliced precursors that are generated in the prp22-1 mutant.

In the case of the mud1s and nam $8 \Delta$ mutants for which the corresponding proteins are part of the U1 snRNP and are involved in the first step of splicing (Liao et al. 1993; Gottschalk et al. 1998), we found similarly that inactivation of 


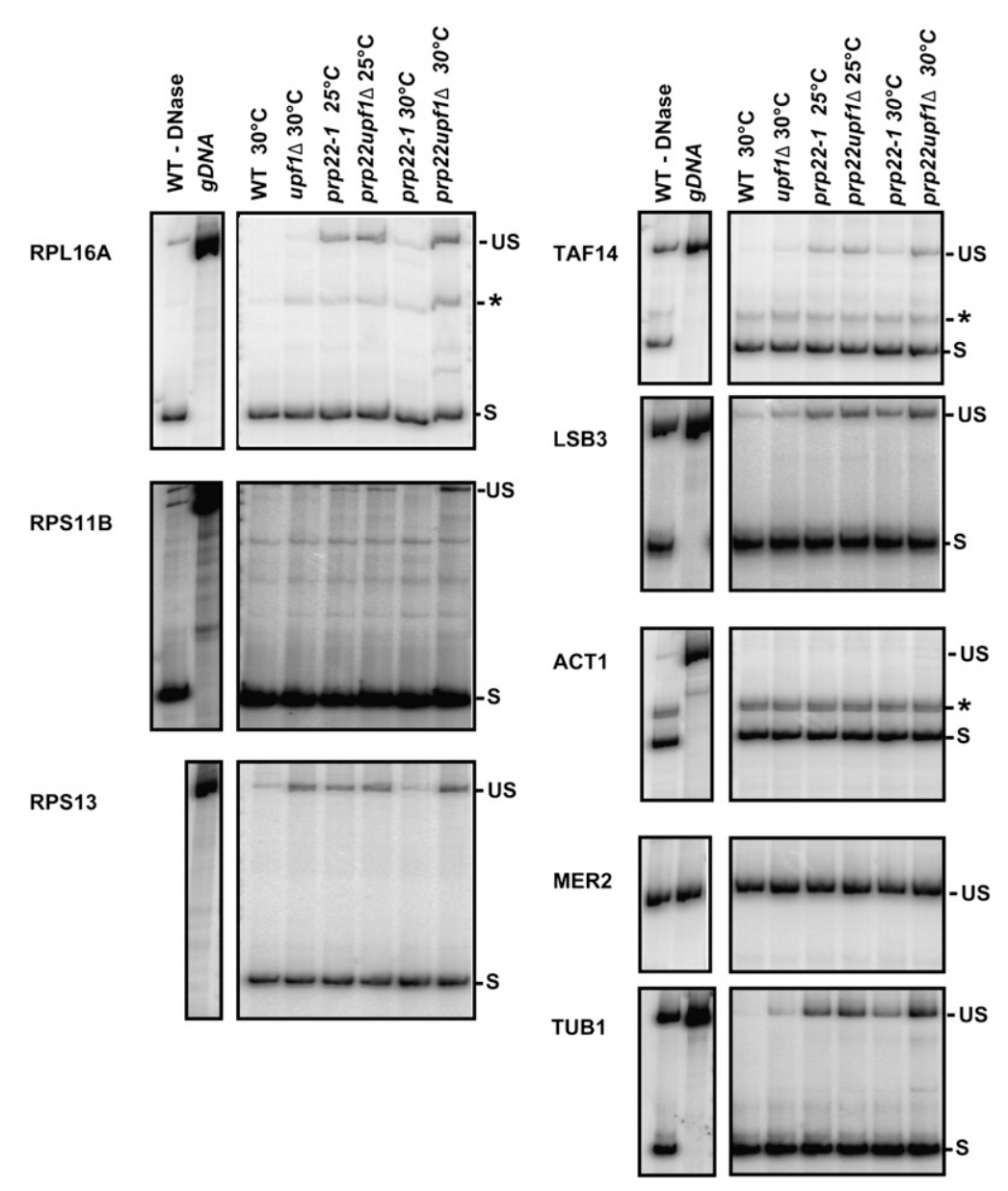

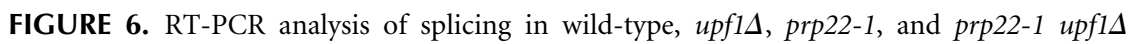
mutants; legend as in Figures 4 and 5.

Upflp in these mutants increased the accumulation of unspliced forms of RPL16A and RPS11A (Fig. 7). In addition, TAF14 unspliced pre-mRNAs were observed only

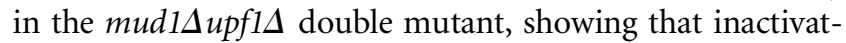
ing the NMD can reveal splicing defects specific to the deletion of MUD1, which are not observed when Nam8p is inactivated. In contrast, inactivation of Upflp did not reveal nor exacerbate the accumulation of unspliced precursors for the LSB3, ACT1, MER2, and TUB1 transcripts. As observed for the previous mutants, inactivation of Mudlp or of Nam8p had little effect on the accumulation of unspliced RPS13 transcripts, which are already abundant in the upf1A mutant. Thus, similarly to what we found for the second

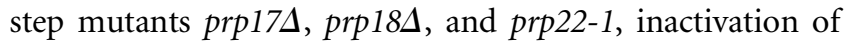
Upflp can reveal some of the splicing defects that are otherwise masked by NMD in first step mutants.

\section{Genetic interactions between upf1s and splicing mutants}

Following the previous observations, we could not exclude the possibility that the increase of unspliced precursors observed in the double mutants might have been caused by synergistic growth defects. While generating the double mutants combining the upfis deletion with the splicing mutants, we found, to the contrary, that the double mutants in which the prp174 or prp22-1 mutation are combined with the upfls deletion exhibited a better growth rate than those of the single prp174 or prp22-1 mutant (Fig. 8). Inactivating Upflp was, however, not sufficient to rescue the temperature-sensitive phenotype of the prp22-1 mutant (data not shown). Therefore, inactivation of NMD partially suppresses the growth defects associated with the prp17s or prp22-1 splicing mutants. This is in contrast to the deletion of Prp18p, which shows a synthetic growth defect when combined with deletion of Upflp (Fig. 8). This result is reminiscent of that observed for the splicing/nuclear retention factor $\mathrm{BBP} / \mathrm{ScSF} 1$, for which inactivation of NMD results in a synthetic lethal phenotype (Rutz and Seraphin 2000). The reduced growth of the prp18supfis double mutant compared with the prp18s mutant might explain why the quantitative effects of NMD inactivation were less pronounced for the prp18s mutant than for the prp17s mutant (Figs. 1-3).

We also monitored the growth rates of nam $8 \Delta, \operatorname{mud} 1 \Delta$, upf $1 \Delta$, and corresponding double mutants, but neither of the single or double mutants exhibited a significant growth defect on plates (data not shown). Thus, the positive genetic interactions observed between the $u p f 1 \Delta$ strain and splicing mutants seem to be specific to prp22-1 and prp17A. The growth suppression phenotypes observed between the

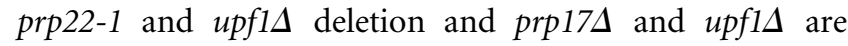
rather surprising, given the fact that previous work had shown that the combination of a mutation of the splicing/ retention factor BBPS/ScSF1 and NMD inactivation results in a synthetic lethal phenotype (Rutz and Seraphin 2000) and given the negative genetic interaction observed between the upfls deletion and the prp18s mutant (Fig. 8). However, this result can be explained by considering the splicing of a specific group of transcripts that might be rate limiting for growth. It is possible that the absence of Prp17p or the partial inactivation of Prp22p at permissive temperature results in reduced production of these rate-limiting spliced mRNAs. Inactivation of NMD in this context might increase the level of the corresponding unspliced RNAs, which might ultimately increase the production of spliced 


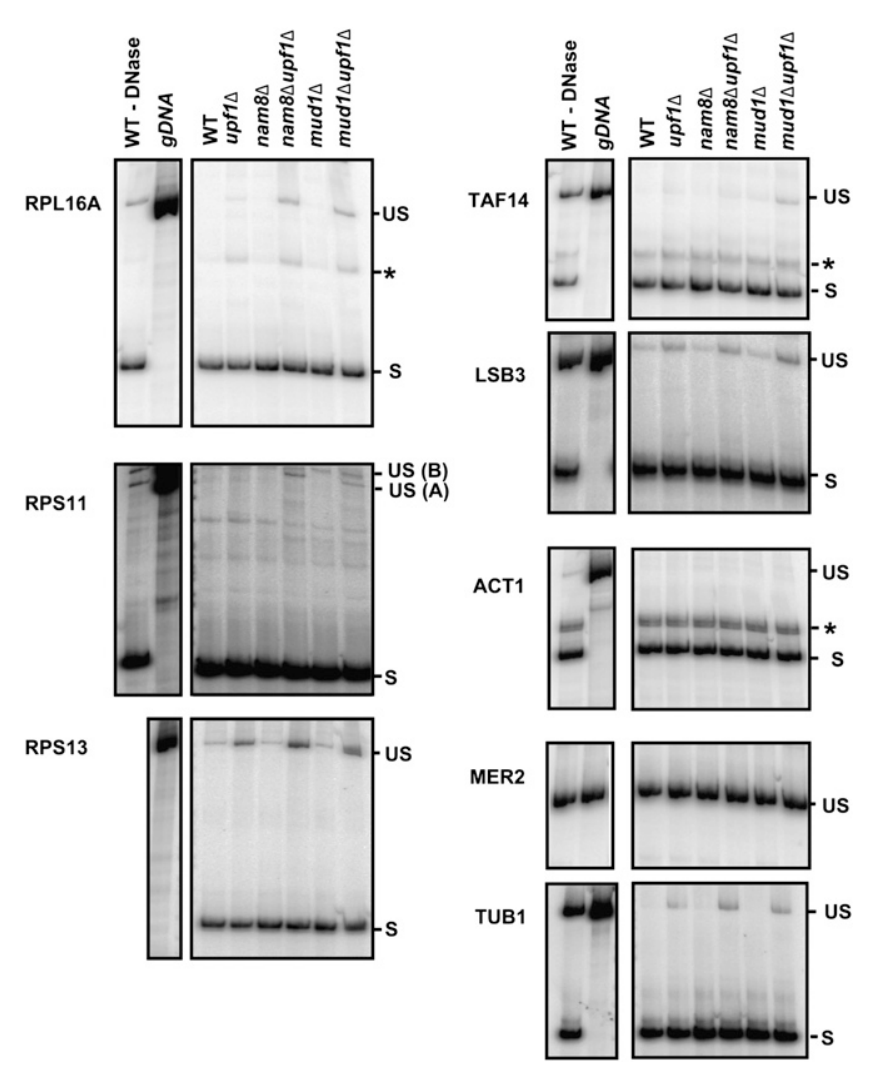

FIGURE 7. RT-PCR analysis of splicing in wild-type, upf1 $1 \Delta$, nam $8 \Delta$, mud1s, nam $8 \Delta u p f 1 \Delta$, and mud1 upfls mutants; legend as in Figures 4 and 5.

mRNAs, because more precursors are available for splicing. A similar model has been proposed to explain the suppression of branchpoint mutations by the Prp16p splicing factor mutations (Burgess et al. 1990). However, we were unable to identify transcripts using tiling arrays for which exonic

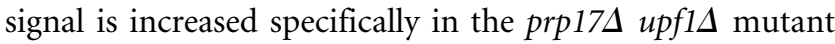

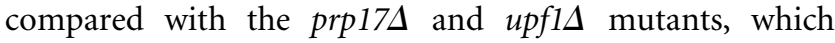
would have indicated increased splicing. Thus, it is possible that the suppression observed is due to other effects (see Discussion). Regardless of the interpretation, these results identify a positive genetic interaction between an NMD mutant and several splicing factor mutants.

\section{DISCUSSION}

In this study we have shown that NMD can degrade a fraction of unspliced precursors generated by deletion or inactivation of the spliceosome components Prp17p, Prp18p, Prp22p, Mud1p, and Nam8p. Thus, NMD inactivation enhances the quantitative effects of several splicing factor mutations and also reveals transcripts that were thought to be unaffected by these splicing factors. Interestingly, our analysis reveals that the prp17A and prp18s mutants exhibit accumulation of unspliced precursors (Figs. 4, 5). The Prp17p and Prp18p spliceosome components are thought to be involved specifically in the second step of splicing (Jones et al. 1995; Umen and Guthrie 1995), yet they exhibit a first step defect as shown by the accumulation of unspliced precursors in vivo (Figs. $4,5)$. This observation could be explained by a hitherto unidentified involvement of these factors in the first step of splicing. Indeed, Prp17p was found to be associated biochemically with pre-first step C-complex in mammalian cells (Bessonov et al. 2008) and in pre-catalytic spliceosomes in yeast (Sapra et al. 2008). This association with pre-first step complexes might provide an explanation for the first-step defect observed for strains lacking Prp17p in vivo. Alternatively, it is possible that disrupting second-step splicing factors may shift the equilibrium of spliceosome conformations toward a first step specific conformation, as suggested by recent genetic analyses (Konarska et al. 2006; Liu et al. 2007). This equilibrium shift might then favor the reversal of the first step in vivo, resulting in the reverse production of unspliced precursors. This hypothesis, although complex, is supported by the observation that both steps of pre-mRNA splicing are reversible, at least in vitro (Tseng and Cheng 2008). Finally, it is possible that the unspliced precursor phenotype observed in these secondstep mutants is indirect and due to the sequestration of a limiting first step splicing factor in spliceosomes stalled prior to the second catalytic step in vivo.

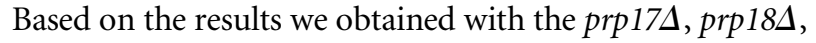
nam8s, mud1s, and prp22-1 mutants, it is puzzling that a previous analysis of the prp2-1 splicing mutant did not result in the stabilization of unspliced pre-mRNAs upon inactivation of NMD (Bousquet-Antonelli et al. 2000). It is possible that unspliced precursors in the prp2-1 mutant are

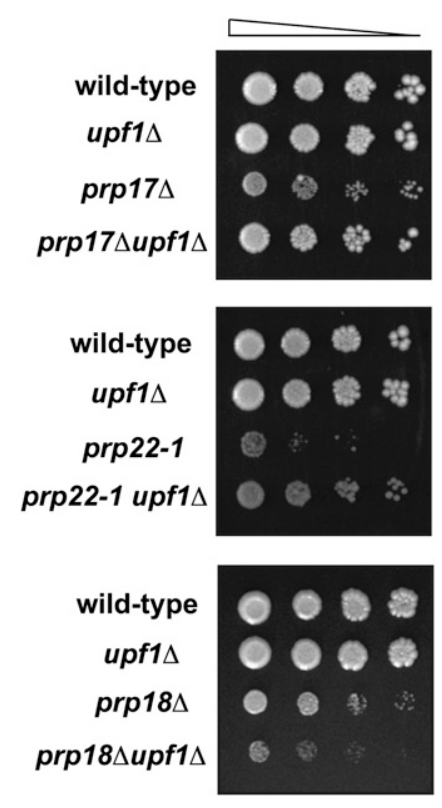

FIGURE 8. Growth of wild-type, upfis, splicing mutants and the corresponding double mutants. Plates were incubated for $4 \mathrm{~d}$ at $25^{\circ} \mathrm{C}$. 
trapped in a pre-catalytic conformation of the spliceosome in the nucleus and specifically degraded by the nuclear exosome because of nuclear retention. Alternatively, it is possible that since the analysis of the prp2-1 splicing mutant was performed on only a few transcripts (Bousquet-Antonelli et al. 2000), some unspliced transcripts generated by the prp2-1 mutant and degraded by NMD may have escaped identification.

Our results show that NMD targets many more unspliced precursors than we previously estimated (Sayani et al. 2008). In natural conditions, some transcripts are spliced with very high efficiency, and therefore generate very few unspliced precursors. However, in conditions where splicing is suboptimal due to nonessential splicing

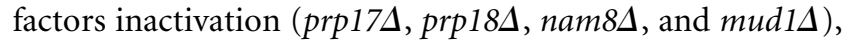
or because of partial inactivation of an essential splicing factor at a permissive temperature (prp22-1), unspliced precursors escape the spliceosome and are degraded by NMD. This process might compete with splicing, as shown by the partial rescue of the growth defect of the prp17A and prp22-1 mutations by Upflp inactivation (Fig. 6). We do not yet understand the molecular basis for this suppression and why the positive genetic interactions are specific to prp174 and prp22-1. One interpretation is that inactivation of Upflp might increase the growth of these splicing mutants by increasing the level of unspliced precursors of transcripts that are rate limiting for growth, thereby increasing the pool of splicing substrates for these transcripts and possibly the amount of mature mRNA produced. Since pre-mRNA splicing is nuclear, and NMD is cytoplasmicor perinuclear-this would require the shuttling back of unspliced pre-mRNAs to the nucleus. Unfortunately, based on inspection of the tiling arrays, we have not been able to identify transcripts for which spliced transcripts are in-

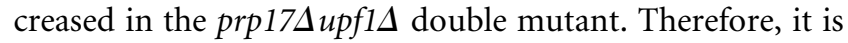
formally possible that these genetic interactions rely on molecular interactions that are unrelated to splicing.

Several studies have investigated the effects of spliceosome mutations in a genome-wide manner to identify transcripts whose splicing requires specific spliceosome components (Clark et al. 2002; Sapra et al. 2004; Pleiss et al. 2007). Conclusions about transcript-specific effects of spliceosome component mutations based on the measurement of precursor accumulation are problematic, since the absence of effect of a mutation on the accumulation of specific unspliced transcripts might have been the result of RNA degradation masking the accumulation of unspliced precursors, as shown in this study. Analysis of the reduction of mature mRNA production is a more informative parameter to measure splicing defects directly in vivo. However, in the case of stable mRNAs, splicing defects do not necessarily result in a reduction of mature mRNAs (Pikielny and Rosbash 1985), making this approach impractical in many cases, especially in thermosensitive mutants for which a rapid shift to a nonpermissive tem- perature is used. Analysis of our data shows that the full extent of the effects of spliceosome mutations can be revealed only when the NMD (or any other RNA degradation system that also affects unspliced RNAs) is inactivated. Some transcripts such as RPL16A seem to be affected by all mutants whenever these are combined with NMD inactivation. However, some other transcripts such as TUB1 seem to be affected by only a subset of mutants. Thus, splicing factor mutations seem to affect the splicing of genes to different extents, even when taking into account the effect of RNA degradation by NMD. We emphasize that our study may not have revealed the full extent of the phenotypes associated with the splicing mutants analyzed here because some other RNA degradation systems such as the nuclear exosome may also degrade some transcripts unaffected by NMD, as shown previously (BousquetAntonelli et al. 2000). Even when considering this possibility, our results underscore the importance of RNA surveillance in the quality control of splicing, as a large fraction of unspliced precursors generated by splicing defects are eliminated by RNA degradation.

\section{MATERIALS AND METHODS}

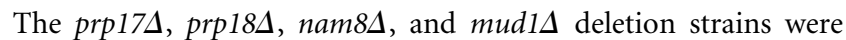
obtained form Open Biosystems. The prp22-1 mutant strain was from Vijayraghavan et al. (1989). Disruption of UPF1 in splicing mutant background was performed using a PCR-based knockout strategy using the HIS marker (Longtine et al. 1998). Analysis of RNAs extracted from the single and double mutant strains by tiling arrays was performed as described (Sayani et al. 2008). Three independent biological replicates were obtained for each single or double mutant, except for the wild-type and upfis strains for which four biological replicates were used (Sayani et al. 2008). Tiling array data are accessible in the GEO database (accession numbers GSE11621 and GSE18288). Tiling array signals were analyzed in MATLAB. Probe signals for the biological replicates for each strain were quantile normalized and averaged. The $\log 2$ of the ratio of the average of for each probe (perfectmatchmismatch) was calculated for each strain comparison. Probes that hybridize 12-nt upstream of and 12-nt downstream from intronic regions were retained for intronic signal analysis. Z-scores were calculated in Matlab and are provided in Supplemental Table 1. Hierarchical clustering analysis using the average method was performed in JMP. Northern blot analysis was performed as described (Sayani et al. 2008). RT-PCR analysis was performed after DNase-I treatment of total RNA samples. A total of $40 \mu \mathrm{g}$ of RNA was treated with $10 \mu \mathrm{L}$ of Ambion RNase-free DNase I in a $100 \mu \mathrm{L}$ reaction at $37^{\circ} \mathrm{C}$ for $1 \mathrm{~h}$. The DNase-treated RNA samples were purified by phenol-chloroform extraction and precipitation and resuspended in $20 \mu \mathrm{L}$ of water. Reverse transcription was performed using random hexamers. A total of $5 \mu \mathrm{g}$ of DNase-treated RNA was incubated with $1 \mu \mathrm{L}$ of random primers $(50 \mathrm{ng} / \mu \mathrm{L})$ in $12 \mu \mathrm{L}$. The reaction was heated to $65^{\circ} \mathrm{C}$ for $5 \mathrm{~min}$ and chilled on ice. Reverse transcription was performed using M-MLV Reverse Transcriptase in a $20 \mu \mathrm{L}$ reaction for $50 \mathrm{~min}$ at $42^{\circ} \mathrm{C}$. The reactions were incubated at $85^{\circ} \mathrm{C}$ for $5 \mathrm{~min}$ to heat inactivate the reverse-transcriptase and treated with $\mathrm{RNase} H$ for 
$20 \mathrm{~min}$ at $37^{\circ} \mathrm{C}$. Two microliters of reverse transcription reactions were used for PCR amplification using standard procedure and including $\left[\alpha^{32}-\mathrm{P}\right] \mathrm{dCTP}$ for detection. Products were fractionated on polyacrylamide gels and the dried gels were exposed for phosphorimaging. The oligonucleotides used to amplify PCR products used for in vitro transcription and generation of riboprobes for Northern blots were the following:

RPL16A forward: GTCTGTTGAACCAGTTGTTGTC; RPL16A reverse: GATCAATTAACCCTCACTAAAGGAGCAGTA GCATTAGCAGAGGCA;

RPS11B forward: AGGAAACGTATCAAGCCAGGGA; RPS11B reverse: GATCAATTAACCCTCACTAAAGGTGGGGAG ACGTGGACTGGG;

RPS13 forward: ATGGGTCGTATGCACAGT; and RPS13 reverse: GATCAATTAACCCTCACTAAAGGCCAGTTTG

\section{GTGGTAAGACAGCAAC.}

The oligonucleotides for RT-PCR analysis were the following:

TUB1 forward: CGCCACCCAAGATCTGTAAACT; TUB1 reverse: ACCAATCTGACAACCAGCTTGACC; TAF14 forward: GTGACCTCGGAGCTGACTGATATT; TAF14 reverse: TCTATACTCCATTGACGAACAGGA; RPL16A forward: ACGGAAGGTGAAGAACAAATCGAG; RPL16A reverse: AGCAACAACGGAAGCTAAACGACC; RPS11B forward: CCACTGAATTAACTGTTCAATCTGAAAGAGC; RPS11B reverse: GGTTCTCTTGGAAGTCTTGACCTTTGG; ACT1 forward: AGAATAGGATCTTCTACTACATCAGCTT; ACT1 reverse: ACGATAGATGGGAAGACAGCACGA; RPS13 forward: AAATGGGTCGTATGCACAGT;

RPS13 reverse: AGGACAACTTGAACCAAGCTGGAG; MER2 forward: GCTACTGGAACAAGATGCTGCTACGA; MER2 reverse: CGTCGCCTTTCGATAACATTGCTG; LBS3 forward: TCCAAGGAGTTTAAAGAGTGAGACA; and LBS3 reverse: AACGTCTGGAGGAATGACTTGGTC.

\section{SUPPLEMENTAL MATERIAL}

Supplemental material can be found at http://www.rnajournal.org.

\section{ACKNOWLEDGMENTS}

We thank M. Janis for preliminary analysis of the prp17 mutant arrays. This work was supported by National Institutes of Health Grant No. GM61518 and American Cancer Society Grant No. RSG-06-040 (to G.C.). T.K. was supported by USPHS National Research Service Award No. GM07104.

Received May 16, 2009; accepted September 15, 2009.

\section{REFERENCES}

Behm-Ansmant I, Kashima I, Rehwinkel J, Sauliere J, Wittkopp N, Izaurralde E. 2007. mRNA quality control: An ancient machinery recognizes and degrades mRNAs with nonsense codons. FEBS Lett 581: 2845-2853.

Bessonov S, Anokhina M, Will CL, Urlaub H, Lührmann R. 2008. Isolation of an active step I spliceosome and composition of its RNP core. Nature 452: 846-850.
Bousquet-Antonelli C, Presutti C, Tollervey D. 2000. Identification of a regulated pathway for nuclear pre-mRNA turnover. Cell 102: 765-775.

Burgess S, Couto JR, Guthrie C. 1990. A putative ATP binding protein influences the fidelity of branchpoint recognition in yeast splicing. Cell 60: 705-717.

Chanfreau G, Legrain P, Dujon B, Jacquier A. 1994. Interaction between the first and last nucleotides of pre-mRNA introns is a determinant of $3^{\prime}$ splice site selection in S. cerevisiae. Nucleic Acids Res 22: 1981-1987.

Chang YF, Imam JS, Wilkinson MF. 2007. The nonsense-mediated decay RNA surveillance pathway. Annu Rev Biochem 76: 51-74.

Clark TA, Sugnet CW, Ares M Jr. 2002. Genomewide analysis of mRNA processing in yeast using splicing-specific microarrays. Science 296: 907-910.

Gottschalk A, Tang J, Puig O, Salgado J, Neubauer G, Colot HV, Mann M, Seraphin B, Rosbash M, Lührmann R, et al. 1998. A comprehensive biochemical and genetic analysis of the yeast U1 snRNP reveals five novel proteins. RNA 4: 374-393.

He F, Peltz SW, Donahue JL, Rosbash M, Jacobson A. 1993. Stabilization and ribosome association of unspliced pre-mRNAs in a yeast upf1- mutant. Proc Natl Acad Sci 90: 7034-7038.

Hilleren PJ, Parker R. 2003. Cytoplasmic degradation of splicedefective pre-mRNAs and intermediates. Mol Cell 12: 1453-1465.

Isken O, Maquat LE. 2008. The multiple lives of NMD factors: Balancing roles in gene and genome regulation. Nat Rev Genet 9: 699-712.

Jaillon O, Bouhouche K, Gout JF, Aury JM, Noel B, Saudemont B, Nowacki M, Serrano V, Porcel BM, Segurens B, et al. 2008. Translational control of intron splicing in eukaryotes. Nature 451: 359-362.

Jones MH, Frank DN, Guthrie C. 1995. Characterization and functional ordering of Slu7p and Prp17p during the second step of pre-mRNA splicing in yeast. Proc Natl Acad Sci 92: 9687-9691.

Konarska MM, Vilardell J, Query CC. 2006. Repositioning of the reaction intermediate within the catalytic center of the spliceosome. Mol Cell 21: 543-553.

Lareau LF, Inada M, Green RE, Wengrod JC, Brenner SE. 2007. Unproductive splicing of SR genes associated with highly conserved and ultraconserved DNA elements. Nature 446: 926-929.

Liao XC, Tang J, Rosbash M. 1993. An enhancer screen identifies a gene that encodes the yeast U1 snRNP A protein: Implications for snRNP protein function in pre-mRNA splicing. Genes \& Dev 7: 419-428.

Liu L, Query CC, Konarska MM. 2007. Opposing classes of prp8 alleles modulate the transition between the catalytic steps of premRNA splicing. Nat Struct Mol Biol 14: 519-526.

Longtine MS, McKenzie A 3rd, Demarini DJ, Shah NG, Wach A, Brachat A, Philippsen P, Pringle JR. 1998. Additional modules for versatile and economical PCR-based gene deletion and modification in Saccharomyces cerevisiae. Yeast 14: 953-961.

Mitrovich QM, Anderson P. 2000. Unproductively spliced ribosomal protein mRNAs are natural targets of mRNA surveillance in C. elegans. Genes \& Dev 14: 2173-2184.

Ni JZ, Grate L, Donohue JP, Preston C, Nobida N, O'Brien G, Shiue L, Clark TA, Blume JE, Ares M Jr. 2007. Ultraconserved elements are associated with homeostatic control of splicing regulators by alternative splicing and nonsense-mediated decay. Genes \& Dev 21: 708-718.

Pikielny CW, Rosbash M. 1985. mRNA splicing efficiency in yeast and the contribution of nonconserved sequences. Cell 41: 119-126.

Pleiss JA, Whitworth GB, Bergkessel M, Guthrie C. 2007. Transcript specificity in yeast pre-mRNA splicing revealed by mutations in core spliceosomal components. PLoS Biol 5: e90. doi: 10.1371/ journal.pbio.0050090.

Rutz B, Seraphin B. 2000. A dual role for BBP/ScSF1 in nuclear premRNA retention and splicing. EMBO J 19: 1873-1886.

Sapra AK, Arava Y, Khandelia P, Vijayraghavan U. 2004. Genomewide analysis of pre-mRNA splicing: Intron features govern the 
requirement for the second step factor, Prp17 in Saccharomyces cerevisiae and Schizosaccharomyces pombe. J Biol Chem 279: 5243752446.

Sapra AK, Khandelia P, Vijayraghavan U. 2008. The splicing factor Prp17 interacts with the U2, U5 and U6 snRNPs and associates with the spliceosome pre- and post-catalysis. Biochem J 416: 365-374.

Sayani S, Janis M, Lee CY, Toesca I, Chanfreau GF. 2008. Widespread impact of nonsense-mediated mRNA decay on the yeast intronome. Mol Cell 31: 360-370.

Scherrer FW Jr, Spingola M. 2006. A subset of Merlp-dependent introns requires Bud13p for splicing activation and nuclear retention. RNA 12: 1361-1372.
Tseng CK, Cheng SC. 2008. Both catalytic steps of nuclear pre-mRNA splicing are reversible. Science 320: 1782-1784.

Umen JG, Guthrie C. 1995. Prp16p, Slu7p, and Prp8p interact with the $3^{\prime}$ splice site in two distinct stages during the second catalytic step of pre-mRNA splicing. RNA 1: 584-597.

Vijayraghavan U, Parker R, Tamm J, Iimura Y, Rossi J, Abelson J, Guthrie C. 1986. Mutations in conserved intron sequences affect multiple steps in the yeast splicing pathway, particularly assembly of the spliceosome. EMBO J 5: 1683-1695.

Vijayraghavan U, Company M, Abelson J. 1989. Isolation and characterization of pre-mRNA splicing mutants of Saccharomyces cerevisiae. Genes \& Dev 3: 1206-1216. 

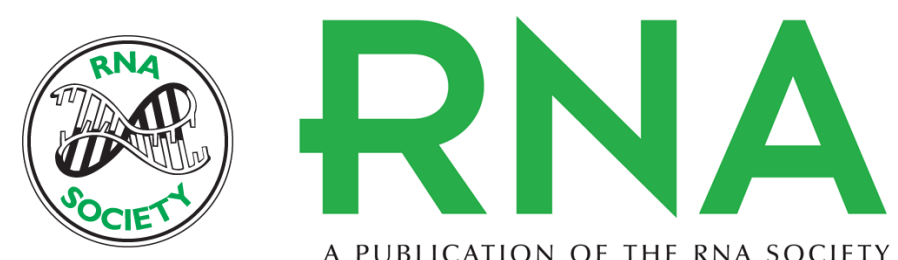

A PUBLICATION OF THE RNA SOCIETY

\section{Nonsense-mediated mRNA decay mutes the splicing defects of spliceosome component mutations}

Tadashi Kawashima, Matteo Pellegrini and Guillaume F. Chanfreau

RNA 2009 15: 2236-2247 originally published online October 22, 2009

Access the most recent version at doi:10.1261/rna.1736809

Supplemental
Material http://rnajournal.cshlp.org/content/suppl/2009/10/20/rna.1736809.DC1

References This article cites 31 articles, 13 of which can be accessed free at: http://rnajournal.cshlp.org/content/15/12/2236.full.html\#ref-list-1

License

Email Alerting Receive free email alerts when new articles cite this article - sign up in the box at the Service top right corner of the article or click here. 